ses Fundortes $\left(27 \cdot 10^{-4}\right)$ als auch die Verhältnisse für die Gläser Ata $\left(20 \cdot 10^{-4}\right)$ und Buonim $\left(18 \cdot 10^{-4}\right)$ stimmen damit recht gut überein. Da alle Tektite mit Ausnahme derjenigen von Indonesien, Kambodscha und Annam Ni/Fe-Verhältnisse zwischen 5 und $10 \cdot 10^{-4}$ haben, ist das Verhältnis von etwa $20 \cdot 10^{-4}$ für die Tektite der Elfenbeinküste sehr charakteristisch. Daß auch die $\mathrm{Ni} / \mathrm{Fe}-$ Verhältnisse für die Gläser Ata und Buonim bei diesem Wert liegen, ist für die Diskussion der Herkunft der Elfenbeinküste-Tektite von entscheidender Bedeutung.

Die in Tab. 2 gezeigten $\mathrm{Cr} / \mathrm{Ni}-$ Verhältnisse sind untereinander sehr ähnlich; sie liegen tiefer als die von TAYlor $^{11}$ für Australite $(2,65)$ und von CHAO ${ }^{12}$ für Bediasite $(2,77)$ angegebenen.

Somit bestätigt auch der spurenanalytische Befund den ursächlichen Zusammenhang zwischen BosumtwiGläsern und Elfenbeinküste-Tektiten, der schon oben durch die übereinstimmenden K-Ar-Alter die erste experimentelle Stütze erhielt.

11 S. R. TAylor, Geochim. Cosmochim. Acta 26, 2, 685 [1962].

12 E.C.T.Cнно, Tektites, University Chicago Press 1963, S. 51.
Es handelt sich also offenbar bei den Beziehungen zwischen Bosumtwi-Gläsern und Elfenbeinküste-Tektiten um einen ähnlichen Fall, wie er früher vom Nördlinger Ries und den Moldaviten beschrieben wurde (Gentner et al. ${ }^{13}$, Schüller et al. ${ }^{9}$ ). In beiden Fällen dürfte ein ähnlich großartiges Naturereignis, wahrscheinlich ein Meteoriteneinschlag, als Ursache anzusehen sein.

Eine ausführliche Darstellung wird an anderer Stelle gemeinsam mit den Professoren TH. Monod und A. F. J. Smit erfolgen, denen wir die Wiederauffindung der Gläser verdanken. Dabei soll auch eine detaillierte geologische und mineralogische Beschreibung erfolgen.

Der Kwame-Nkrumah-Universität in Kumasi und besonders dem Vicechancellor Dr. R. P. BAffour sind wir für die gastliche Aufnahme und die großzügige Unterstützung unserer Exkursionen zu besonderem Dank verpflichtet.

13 W. Gentner, H. J. Lippolt u. O. A. Schaeffer, Geochim. Cosmochim. Acta 27, 191 [1962].

\section{Negative Ionen aus dem Hochfrequenzfunken zwischen Metallelektroden und ihre Verwendbarkeit zu Analysen}

\section{Von K. D. Schuy, J. Franzen und H. Hintenberger}

Max-Planck-Institut für Chemie (Otto-Hahn-Institut), Mainz (Z. Naturforschg. 19 a, 153-155 [1964] ; eingeg. am 20. Dezember 1963)

\section{Massenspektren negativer Ionen}

Der Hochfrequenzfunke zwischen Metallelektroden bildet neben positiven auch negative Ionen, die mit einem doppelfokussierenden Massenspektrographen auf einer Photoplatte nachgewiesen werden können. Die Spektren sind sehr linienreich bis weit über die Masse 100 hinaus und enthalten viele Multipletts. Spektren aus Elektroden von verschiedenen Metallen sind in ihrer Linienzusammensetzung sehr ähnlich. Die Linien der Hauptkomponenten des Elektrodenmaterials fehlen oft völlig. Die Identifizierung der Linien negativer Ionen ist ohne Kenntnis der genauen Massen meist nicht möglich.

\section{Linienidentifizierung}

Durch Berechnung der genauen Massen aus den Linienabständen konnten wir im Massenbereich 12 bis 48 die Linienspektren aus verschiedenen Metallelektroden vollständig identifizieren. Im Bereich höherer Massen reicht zur Zeit das Auflösungsvermögen des benutzten

* Abb. 1 und 2 auf Tafel S. 154 a.

1 H. Hintenberger, J. Franzen u. K. D. Schuy, Z. Naturforschg. 18 a, 1236 [1963].

2 W. M. Hickam u. G. G. Sweeney, Rev. Sci. Instr. 34, 783 [1963].
Apparates nicht aus, um zwischen den Elementkombinationen sicher zu unterscheiden. Abb. $1^{*}$ zeigt im Ausschnitt einen Vergleich von Massenspektren negativer Ionen aus verschiedenen Metallproben. In den Tab. 1 und 2 sind die Linien zweier Spektren identifiziert.

\section{Wichtigste Ionenarten}

Tab. 3 zeigt alle bisher sicher identifizierten Ionen. Die Halogene bilden intensitätsstark einatomige negative Ionen. Die Elemente der VI. Hauptgruppe erscheinen einatomar, zweiatomar und als Hydride. Die Elemente der V. Hauptgruppe treten nur als Hydride auf. Der Kohlenstoff aus der IV. Hauptgruppe bildet negativ geladene Polymere mit starker Bevorzugung von $\mathrm{C}_{2}{ }^{-}$, wie sie auch im Hochfrequenzfunken zwischen Graphitelektroden auftreten ${ }^{\mathbf{1}, 2}$. Die Polymere des Kohlenstoffs erscheinen außerdem gekoppelt mit Wasserstoff und den Elementen der V., VI. und VII. Hauptgruppe. Sehr auffällig in allen Spektren sind die Liniengruppen bei den Massen $24\left(\mathrm{C}_{2}^{-}\right), 25\left(\mathrm{C}_{2} \mathrm{H}^{-}\right)$und $26\left(\mathrm{CN}^{-}\right)$, ferner bei den Massen $42\left(\mathrm{CNO}^{-}\right)$und $45\left(\mathrm{CO}_{2} \mathrm{H}^{-}\right)$, die schon früher bei anderen Erzeugungsarten negativer Ionen gefunden, aber noch nicht identifiziert worden waren ${ }^{2-4}$.

\section{Herkunft der Ionen}

Versuche mit verschiedenen Restgasdrucken und verschiedenen $\mathrm{N}_{2}$ - und Ar-Füllungen im Druckbereich von

3 M.v. Ardenne, K. Steinfelder u. R. Tümmler, Angew. Chem. 73, 136 [1961].

4 R. E. Honig, Advances in Mass Spectrometry Vol. 2, Pergamon Press, London 1963. 


\begin{tabular}{|c|c|c|c|}
\hline Gemessene Masse & Wahre Masse & Ion & $\begin{array}{l}\text { Relative } \\
\text { Häufigkeit }\end{array}$ \\
\hline $\begin{array}{l}\text { Bezugslinie } \\
13,00795 \\
\text { Bezugslinie } \\
15,01097 \\
15,02349 \\
15,99391 \\
\text { Bezugslinie } \\
17,00218 \\
17,99894 \\
\text { Bezugslinie } \\
\text { Bezugslinie } \\
25,00761 \\
\text { Bezugslinie } \\
27,00610 \\
27,02371 \\
\text { Bezugslinie } \\
31,99013 \\
32,97934 \\
32,99837 \\
34,96951 \\
\text { Bezugslinie } \\
36,96480 \\
37,00638 \\
39,99530 \\
41,00268 \\
\text { Bezugslinie } \\
44,99751 \\
\text { Bezugslinie } \\
47,99881\end{array}$ & $\begin{array}{l}12,00000 \\
13,00783 \\
14,01565 \\
15,01090 \\
15,02348 \\
15,99491 \\
16,01872 \\
17,00274 \\
17,99916 \\
18,99840 \\
24,00000 \\
25,00782 \\
26,00307 \\
27,00643 \\
27,02348 \\
31,97207 \\
31,98983 \\
32,97990 \\
32,99766 \\
34,96885 \\
36,00000 \\
36,96590 \\
37,00783 \\
39,99492 \\
41,00274 \\
41,99799 \\
44,99766 \\
47,96699 \\
48,00000 \\
63 \\
64 \\
79 \\
80 \\
96 \\
97\end{array}$ & $\begin{array}{l}\mathrm{C}^{-} \mathrm{CH}^{-} \\
\mathrm{CH}_{2}^{-} \\
\mathrm{NH}^{-} \\
\mathrm{CH}_{3}^{-} \\
\mathrm{O}^{-} \\
\mathrm{NH}_{2}^{-} \mathrm{OH}^{-} \\
{ }^{18} \mathrm{O}^{-}{ }^{-} \\
\mathrm{C}_{2}^{-} \mathrm{F}^{-} \\
\mathrm{CN}^{-}{ }^{\mathrm{C}_{2} \mathrm{H}^{-}} \\
{ }^{13} \mathrm{CN}_{2} \mathrm{H}_{3}^{-} \\
\mathrm{S}^{-} \\
\mathrm{O}_{2}^{-}{ }^{\mathrm{SH}^{-}} \\
\mathrm{O}_{2} \mathrm{H}^{-} \\
\mathrm{Cl}^{-} \\
\mathrm{C}_{3}^{-} \mathrm{Cl}^{-} \\
\mathrm{C}_{3} \mathrm{H}^{-} \\
\mathrm{C}_{2} \mathrm{O}^{-} \\
\mathrm{C}_{2} \mathrm{OH}^{-} \\
\mathrm{CNO}^{-} \\
\mathrm{CO}_{2} \mathrm{H}^{-} \\
\mathrm{SO}^{-} \\
\mathrm{C}_{4}^{-}{ }^{-} \\
\mathrm{CuH}^{-} \\
\mathrm{Cu}^{-} \\
\mathrm{CuOH}^{-} \\
\mathrm{CuO}^{-} \\
\mathrm{CuO}_{2} \mathrm{H}^{-}\end{array}$ & $\begin{array}{r}100 \\
10 \\
3 \\
1 \\
3 \\
3000 \\
1 \\
1000 \\
10 \\
30 \\
300 \\
30 \\
300 \\
3 \\
1 \\
3 \\
30 \\
1 \\
3 \\
3000 \\
10 \\
1000 \\
1 \\
10 \\
3 \\
30 \\
30 \\
1 \\
10 \\
10000 \\
1 \\
1000 \\
3 \\
100 \\
10\end{array}$ \\
\hline
\end{tabular}

Tab. 1. Identifizierung der Linien und relative Häufigkeiten negativer Ionen einer Reinstkupferprobe (Nordd e u t s c h e A f finerie). Zur Kontrolle der benutzten Bezugslinien wurden weitere Bestimmungen mit anderen Bezugslinien durchgeführt, welche die gleichen Ergebnisse lieferten.

$1 \cdot 10^{-6}$ bis $2 \cdot 10^{-4}$ Torr in der Ionenquelle ergaben keine meßbaren Intensitätsänderungen der verschiedenen Linien. Dieser Befund macht wahrscheinlich, daß die nachgewiesenen Ionen nicht aus dem Restgas, sondern aus dem Elektrodenmaterial stammen.

\section{Möglichkeiten zur Festkörperanalyse}

Die negativen Ionen aus dem Hochfrequenzfunken können für einen empfindlichen Nachweis der Halogene und der Elemente C, N, O, H, S usw. in Metallen ausgenutzt werden. Die quantitative Analyse des Elektrodenmaterials bereitet jedoch Schwierigkeiten, da die Analysenelemente häufig in jeweils mehreren Verbindungen vorkommen und, zumindest bei normaler Elektronenanlagerung, die Bildungswahrscheinlichkeiten verschiedener negativer Ionenarten verschieden sind ${ }^{3}$. Abb. 2 zeigt ein Beispiel einer Vergleichsanalyse auf die Elemente $\mathrm{Br}$ und J. Dazu wurden Elektroden aus gesintertem „ferrum reductum, p. a." mit Lösungen von $\mathrm{KBr}$ und $\mathrm{KJ}$ in verschiedenen

\begin{tabular}{|c|c|c|c|}
\hline Gemessene Masse & Wahre Masse & Ion & $\begin{array}{l}\text { Relative } \\
\text { Häufigkeit }\end{array}$ \\
\hline $\begin{array}{l}\text { Bezugslinie } \\
13,00768 \\
14,01584 \\
15,02331 \\
\text { Bezugslinie } \\
16,01798 \\
17,00281 \\
\text { Bezugslinie } \\
23,99994 \\
\text { Bezugslinie } \\
26,00315 \\
31,97258 \\
31,99039 \\
32,97975 \\
32,99808 \\
\text { Bezugslinie } \\
36,96519 \\
41,00321 \\
\text { Bezugslinie } \\
44,01277 \\
44,99721 \\
\text { Bezugslinie }\end{array}$ & $\begin{array}{l}12,00000 \\
13,00783 \\
14,01565 \\
15,02348 \\
15,99491 \\
16,01872 \\
17,00274 \\
18,99840 \\
24,00000 \\
25,00782 \\
26,00307 \\
31,97207 \\
31,98983 \\
32,97990 \\
32,99766 \\
34,96885 \\
36,96590 \\
41,00274 \\
41,99799 \\
44,01364 \\
44,99766 \\
48,00000 \\
56 \\
72\end{array}$ & $\begin{array}{l}\mathrm{C}^{-} \\
\mathrm{CH}_{2}^{-} \mathrm{CH}^{-} \\
\mathrm{O}^{-} \mathrm{CH}_{3}^{-} \\
\mathrm{OH}^{-} \mathrm{NH}_{2}^{-} \\
\mathrm{C}_{2}^{-} \mathrm{F}^{-} \\
\mathrm{CN}^{-} \mathrm{C}_{2} \mathrm{H}^{-} \\
\mathrm{O}_{2}^{-} \mathrm{S}^{-} \\
\mathrm{O}_{2} \mathrm{H}^{-} \\
\mathrm{SH}^{-} \\
\mathrm{Cl}^{-} \\
\mathrm{Cl}^{-} \\
\mathrm{CNO}^{-} \mathrm{C}_{2} \mathrm{OH}^{-} \\
\mathrm{CNOH}_{2}^{-} \mathrm{CNO}_{2} \mathrm{H}^{-} \\
\mathrm{C}_{4}^{-} \\
\mathrm{Fe}^{-}{ }_{\mathrm{FeO}}^{-}\end{array}$ & $\begin{array}{r}100 \\
300 \\
30 \\
30 \\
3000 \\
30 \\
3000 \\
3000 \\
300 \\
1000 \\
3000 \\
100 \\
100 \\
30 \\
100 \\
3000 \\
1000 \\
30 \\
3000 \\
30 \\
1000 \\
10 \\
<1 \\
<3\end{array}$ \\
\hline
\end{tabular}

Tab. 2. Identifizierung der Linien und relative Häufigkeiten negativer Ionen einer Reineisenprobe („Low Alloy Steel E“, Nr. 1165, Nat. Bur. Stand. Washington D.C.). Negative Ionen des Eisens oder einer Eisenverbindung konnten nicht nachgewiesen werden. Auch in wesentlich stärker exponierten Aufnahmen traten diese Linien nicht auf.

\begin{tabular}{|c|c|c|c|}
\hline $\mathrm{C}^{-}$ & $\begin{array}{l}\mathrm{CH}^{-} \\
\mathrm{CH}_{2}^{-} \\
\mathrm{CH}_{3}{ }^{-}\end{array}$ & $\mathrm{CN}^{-}$ & $\begin{array}{l}\mathrm{CNO}^{-} \\
\mathrm{CO}_{2} \mathrm{H}^{-} \\
\mathrm{CNOH}_{2}^{-}\end{array}$ \\
\hline $\mathrm{C}_{2}^{-}$ & $\begin{array}{l}\mathrm{C}_{2} \mathrm{H}^{-} \\
\mathrm{C}_{2} \mathrm{H}_{3}^{-}\end{array}$ & $\begin{array}{l}\mathrm{C}_{2} \mathrm{~N}^{-} \\
\mathrm{C}_{2} \mathrm{NH}^{-}\end{array}$ & $\begin{array}{l}\mathrm{C}_{2} \mathrm{O}^{-} \\
\mathrm{C}_{2} \mathrm{OH}^{-} \\
\mathrm{C}_{2} \mathrm{O}_{2} \mathrm{H}_{3}^{-}\end{array}$ \\
\hline $\mathrm{C}_{3}{ }^{-}$ & $\begin{array}{l}\mathrm{C}_{3} \mathrm{H}^{-} \\
\mathrm{C}_{3} \mathrm{H}_{2}^{-}\end{array}$ & $\mathrm{C}_{3} \mathrm{~N}^{-}$ & \\
\hline $\mathrm{C}_{4}{ }^{-}$ & $\begin{array}{l}\mathrm{C}_{4} \mathrm{H}^{-} \\
\mathrm{NH}^{-} \\
\mathrm{NH}_{2}^{-}\end{array}$ & & \\
\hline $\begin{array}{l}\mathrm{O}^{-} \\
\mathrm{O}_{2}^{-}\end{array}$ & $\begin{array}{l}\mathrm{OH}^{-} \\
\mathrm{O}_{2} \mathrm{H}^{-}\end{array}$ & & \\
\hline $\mathrm{F}^{-}$ & & & \\
\hline $\begin{array}{l}\mathrm{S}^{-} \\
\mathrm{Cl}^{-}\end{array}$ & $\mathrm{SH}^{-}$ & & $\mathrm{SO}^{-}$ \\
\hline $\mathrm{Cu}^{-}$ & $\mathrm{CuH}^{-}$ & & $\begin{array}{l}\mathrm{CuO}^{-} \\
\mathrm{CuOH}^{-} \\
\mathrm{CuO}_{2}^{-} \\
\mathrm{CuO}_{2} \mathrm{H}^{-}\end{array}$ \\
\hline
\end{tabular}

Tab. 3. Aufstellung aller in verschiedenen $\mathrm{Cu}-$ und Fe-Spektren identifizierten negativen Ionen.

Konzentrationsverhältnissen getränkt. Die obere Expositionsserie (5 Aufnahmen) stammt von Elektroden, die mit $\mathrm{Br}$ und $\mathrm{J}$ im atomaren Konzentrationsverhältnis 10:1 getränkt waren. Zur Erzeugung der mittleren 
K. D. Schuy, J. Franzen und H. Hintenberger, Negative Ionen aus dem Hochfrequenzfunken zwischen Metallelektroden und ihre Verwendbarkeit zu Analysen (S. 153).

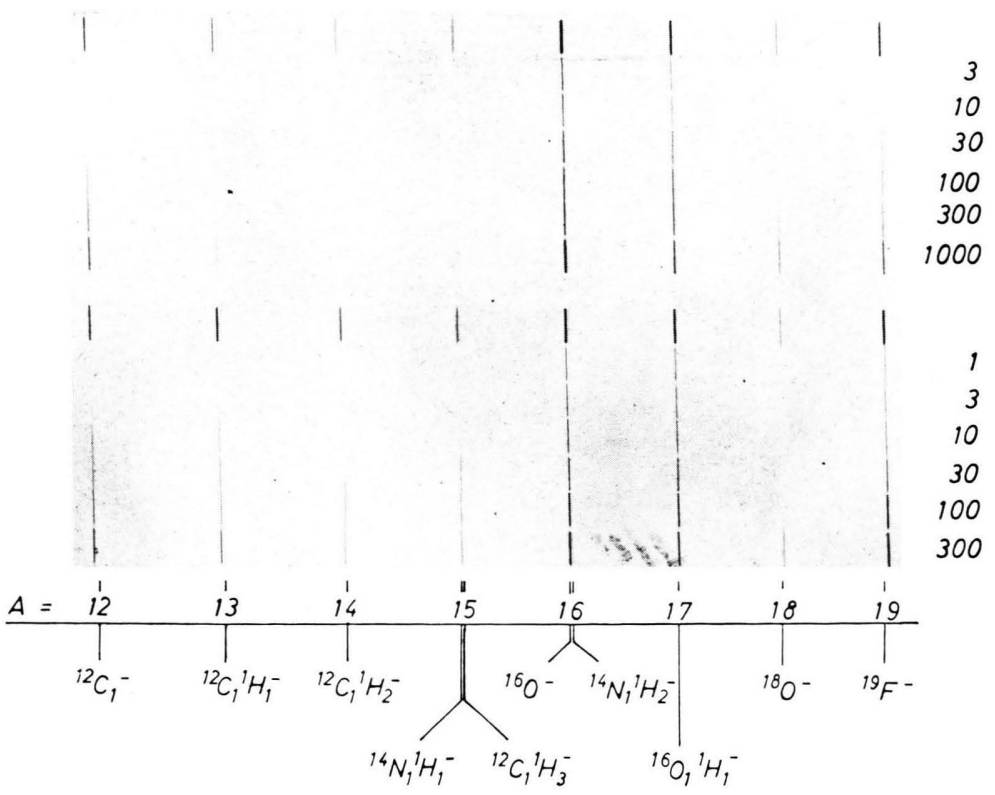

Abb. 1. Ausschnitt aus einer Vergleichsaufnahme negativer Ionen eines Kupferspektrums (oben) und eines Eisenspektrums (unten). Die Zahlen am rechten Rand der Abbildung geben die Expositionsverhältnisse in willkürlichen Einheiten an. Die starken Aufnahmen über jeder Expositionsserie sind Abfunkaufnahmen zur Reinigung der Oberfläche.

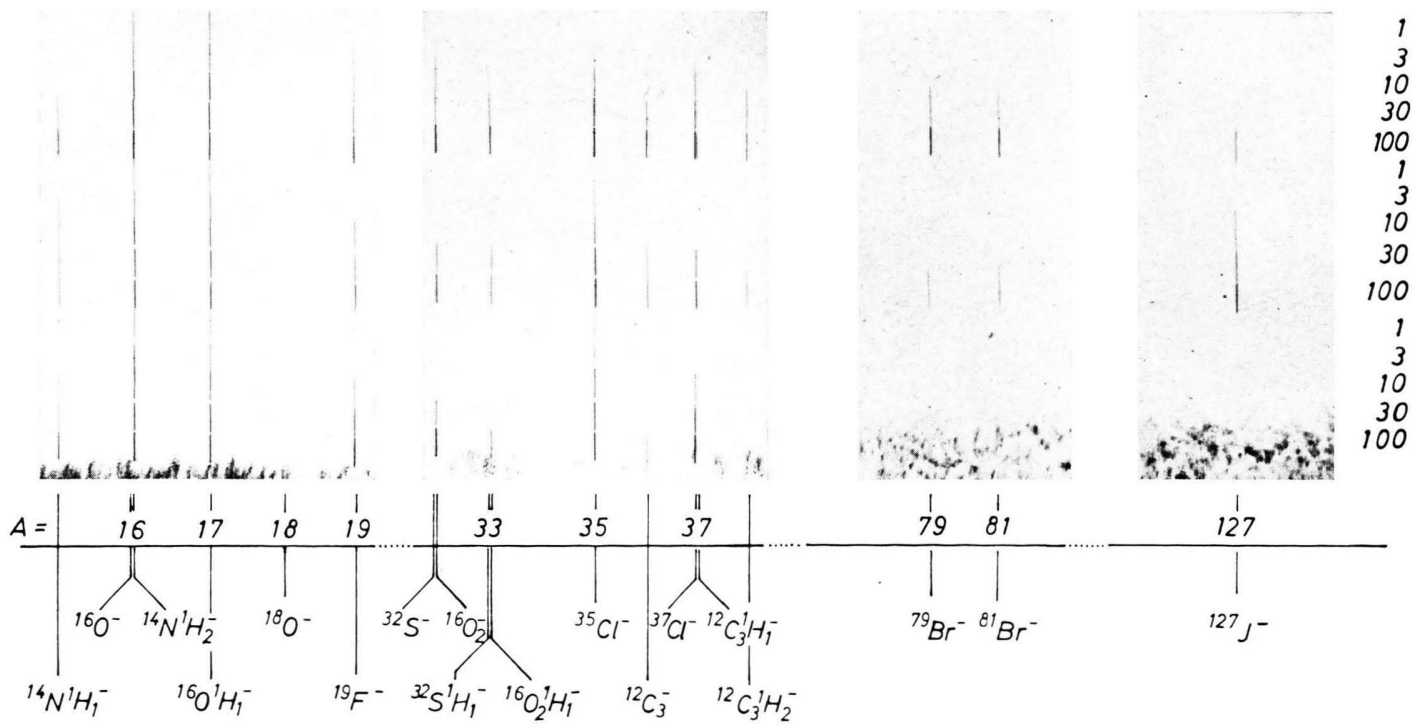

Abb. 2. Ausschnitte aus einer Vergleichsanalyse von Br und J in Proben aus gesintertem „ferrum reductum, p. a.“. Obere 5 Aufnahmen: atomares Tränkungsverhältnis $\mathrm{Br}: \mathrm{J}$ wie $10: 1$. Mittlere 5 Aufnahmen: atomares Tränkungsverhältnis Br: J wie 1 : 10. Untere 5 Aufnahmen: ungetränkte Elektroden. Fluor und Chlor entstammen dem „ferrum reductum, p. a.“. 
Expositionsserie (5 Aufnahmen) wurde das Konzentrationsverhältnis umgekehrt (1:10). Die letzte Expositionsserie (5 Aufnahmen) wurde mit ungetränkten Elektroden gewonnen.

Die Analysenempfindlichkeiten für $\mathrm{Cl}$ und $\mathrm{F}$ sind um zwei bis drei Größenordnungen höher als die für $\mathrm{Br}$ und $\mathrm{J}$, so daß die geringen im „ferrum reductum“ enthaltenen F- und Cl-Spuren bereits zu sehr intensiven Linien führten, die in Abb. 2 ebenfalls wiedergegeben sind. Diese Fluor- und Chlorlinien stammen nicht von Apparateverunreinigungen: Abb. 1 zeigt, daß die $\mathrm{F}^{-}$. Linienintensitäten aus zwei verschiedenen Metallproben sehr verschieden sind.

\section{Zerfallsraten von $\mathrm{H}^{-}$-Ionen im Isochron- Zyklotron auf Grund der Lorentz-Kraft}

\author{
Von O. Böttger und H. Franke
}

AEG-Forschungsinstitut Frankfurt (Main) *

(Z. Naturforschg. 19 a, 155 [1964] ; eingegangen am 21. Dezember 1963)

In zunehmendem Maße interessiert man sich für die Beschleunigung von negativen Ionen in Isochron-Zyklotrons. Dieses Interesse beruht auf der Möglichkeit der vollständigen Extraktion durch eine Stripping-Reaktion in einer Folie oder einem Gasstrahl. Der Zerfall der negativen H-Ionen in Elektronen und Atome bei Energien größer als $10 \mathrm{MeV}$ erfolgt in zunehmendem Maße durch die Abtrennung auf Grund der Lonentz-Kraft. Arbeiten hierzu sind von $\mathrm{W}_{\mathrm{RIGHT}}{ }^{1}$, Allison ${ }^{2}$, JudD ${ }^{3}$ sowie Darewych und Neamton ${ }^{4}$ erschienen.

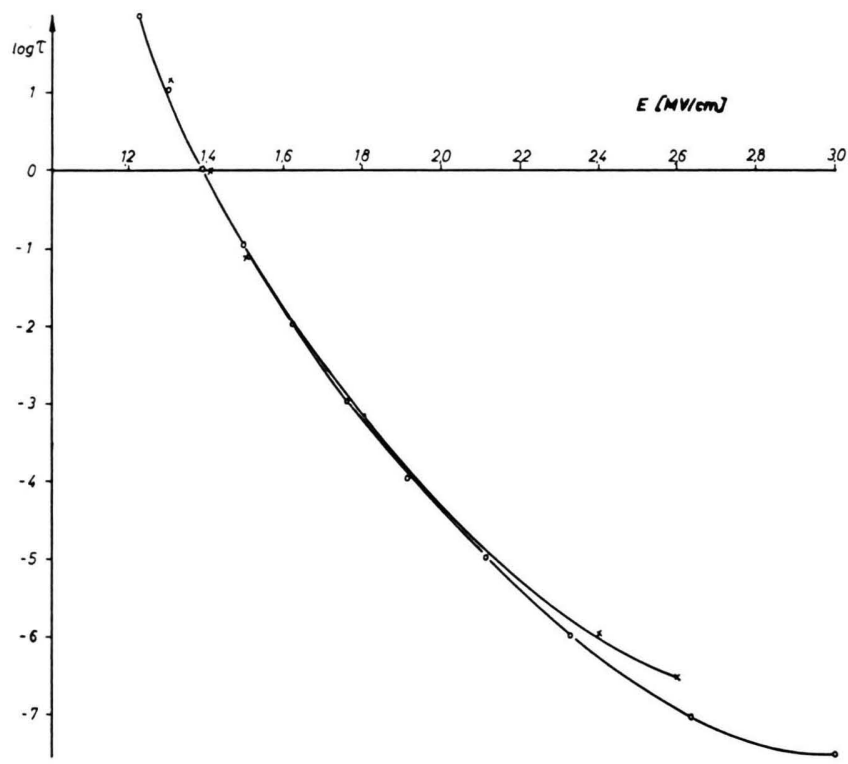

Abb. 1. Vorgegebene Kurve ${ }^{4}(\mathrm{O}-\mathrm{O}-\mathrm{O})$ und Approximation nach Gl. (1) $(\times-\times-x)$. $\ln \tau=(144 c) /(B v)-31,8$.

* Goldsteinstr. 235.

1 B. T. WRIGHT, Arch. Math. Naturvidenskab B. L. IV Nr. 2 [1958], S. 9.

2 S. K. Allison, Rev. Mod. Phys. 30, 1137 [1958].
$\mathrm{Zu}$ einer quantitativen Bestimmung der oben genannten Elemente muß die Konzentration mindestens eines dieser Elemente im Probenmaterial bekannt sein, um Linienintensitäten vergleichen zu können. Wegen der verschiedenen Analysenempfindlichkeiten ist außerdem die Aufnahme von Eichspektren erforderlich.

Rückschlüsse auf die verschiedenen Verbindungsformen der Analysenelemente in den Metallen sind nur sehr schwer zu gewinnen, da im Funken sowohl Dissoziationen als auch Assoziationen auftreten. So zeigen die Spektren aus $\mathrm{NH}_{4} \mathrm{Cl}$-getränkter Kohle sehr intensive Linien für $\mathrm{Cl}^{-}, \mathrm{CN}^{-}$und $\mathrm{CNO}^{-}$, während im Blindversuch mit reiner Kohle diese Linien nur schwach sind.

Wir haben die Kurven von Darewych und Neamton für die Lebensdauer $\tau$ approximiert durch

$$
\ln \tau=\frac{144 c}{B v}-31,8
$$

(s. Abb. 1) $c / v$ : Lichtgeschwindigkeit/Teilchengeschwindigkeit.

Mit dieser Approximation läßt sich der TeilchenVerlust unter Berücksichtigung der Bedingungen im $60^{\circ}$-Thomas-Zyklotron berechnen. Für ein mittleres Feld von 12 bzw. $16 \mathrm{kG}$ und einen Flutter von 0,5 gibt Abb. 2 den Teilchen-Verlust in Abhängigkeit der Energie.

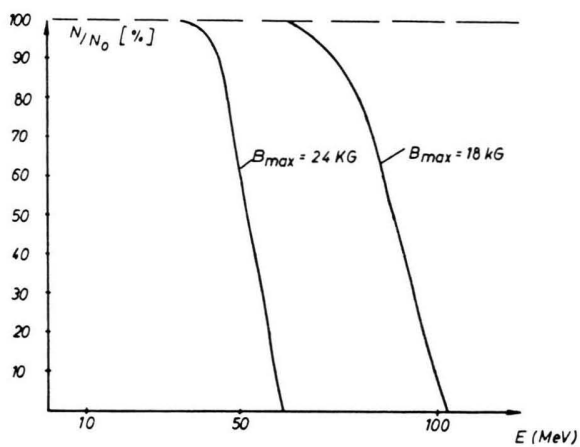

Abb. 2. Verlust an $\mathrm{H}^{-}$-Ionen in Abhängigkeit von der Energie für ein $60^{\circ}$.Thomas-Zyklotron $(f>0,3)$.

Die von uns berechneten Werte liegen günstiger als bisherige Abschätzungen. Dies beruht darauf, daß in den bisherigen Abschätzungen, die sich aus der Endenergie ergebende, nur am Rande der Maschine vorliegende Lebensdauer für die gesamte Maschine in Ansatz gebracht wurde. Da sich die Lebensdauer innerhalb der Maschine von innen nach außen um viele Zehnerpotenzen verändert, muß dieser Ansatz zu wesentlich ungünstigeren Ergebnissen führen.

Eine ausführliche Veröffentlichung erscheint demnächst.

3 D. L. Judd, Nucl. Instr. Methods 18/19, 70 [1962].

4 D. Darewych u. S. M. Neamton, Nucl. Instr. Methods 21 , 247 [1963]. 